\title{
Manejo na prevenção do comportamento suicida dos usuários da Atenção Primária à
}

\section{Saúde: Revisão sistemática}

\author{
Management in the prevention of suicide behavior of users of Primary Health Care: Systematic \\ review
}

Gestión en la prevención del comportamiento suicida de usuarios de Atención Primaria de Salud: Revisión sistemática

Paulla Lopes Ribeiro ORCID: https://orcid.org/0000-0002-9316-9256 Faculdade Dinâmica do Vale do Piranga, Brasil E-mail: paullalopesribeiro@gmail.com

Marco Túlio Vieira de Oliveira ORCID: https://orcid.org/0000-0002-4332-3358 Faculdade Dinâmica do Vale do Piranga, Brasil E-mail: marco.tulio.vieira@hotmail.com

Márcia Farsura de Oliveira ORCID: https://orcid.org/0000-0001-8462-0431 Faculdade Dinâmica do Vale do Piranga, Brasil

E-mail: mmfarsura@yahoo.com.br Marli do Carmo Cupertino ORCID: https://orcid.org/0000-0001-5790-0622 Faculdade Dinâmica do Vale do Piranga, Brasil E-mail: marli.cupertino.vet@gmail.com

\begin{abstract}
Resumo
O comportamento suicida é caracterizado por toda ação na qual o ser humano provoca lesão a si próprio. Levando em consideração que a Atenção Primária à Saúde (APS) é a porta de entrada preferencial dos usuários nos serviços de saúde, esse cenário, passa a exercer um papel crucial na identificação, no manejo e na prevenção do comportamento adscrito. Assim, objetivou revisar, na literatura científica, como se dá o manejo na prevenção do comportamento de autoextermínio dos usuários da APS pela equipe interdisciplinar do serviço de saúde. Para isso, realizou-se uma revisão sistemática, seguindo as diretrizes PRISMA, à partir de estudos brasileiros publicados, no formato de artigo científico original, na base de dados SciELO e LILACS, entre os anos de 2016 e 2021 . Foram recuperados 28 artigos, dos quais 7 foram selecionados de acordo com os critérios de inclusão, para compor a amostra. Verificou-se que apesar da APS possuir estratégias para agir preventivamente no manejo do comportamento voltado ao autoextermínio, dentre essas, a facilidade da criação de vínculos, o acolhimento do paciente, a identificação, avaliação e o acompanhamento regular do indivíduo e de fatores de risco, a possibilidade de acionar fatores protetores e a visita domiciliar, os profissionais ainda se demonstram incapazes e desamparados para exercer o manejo dos seus usuários. Nesse sentido, sugere novos estudos sobre a temática, que envolva propostas e soluções para sanar as dificuldades encontradas por esses profissionais, assim como a capacitação adequada e a elaboração de estratégias para a prevenção desse fenômeno.
\end{abstract}

Palavras-chave: Suicídio; Atenção primária à saúde; Estratégia saúde da família.

\begin{abstract}
Suicidal behavior is characterized by any action in which the human being causes harm to himself. Taking into account that Primary Health Care (PHC) is the preferred gateway for users to health services, this scenario starts to play a crucial role in the identification, management and prevention of the ascribed behavior. Thus, the objective was to review, in the scientific literature, how the prevention of self-extermination behavior of PHC users is carried out by the interdisciplinary team of the health service. For this, a systematic review was carried out, following the PRISMA guidelines, based on Brazilian studies published, in the format of an original scientific article, in the SciELO and LILACS database, between the years 2016 and 2021. 28 articles were recovered, of which 7 were selected according to the inclusion criteria, to compose the sample. It was found that although PHC has strategies to act preventively in the management of behavior aimed at self-extermination, among these, the ease of creating bonds, patient care, identification, assessment and regular monitoring of the individual and of risk factors, the possibility of triggering protective factors and home visits, professionals still demonstrate incapable and helpless to exercise the management
\end{abstract}


of their users. In this sense, it suggests new studies on the subject, involving proposals and solutions to solve the difficulties encountered by these professionals, as well as adequate training and the development of strategies to prevent this phenomenon.

Keywords: Suicide; Primary health care; Family health strategy.

\section{Resumen}

La conducta suicida se caracteriza por cualquier acción en la que el ser humano se cause daño a sí mismo. Teniendo en cuenta que la Atención Primaria de Salud (APS) es la puerta de entrada preferida de los usuarios a los servicios de salud, este escenario comienza a jugar un papel crucial en la identificación, gestión y prevención de la conducta adscrita. Así, el objetivo fue revisar, en la literatura científica, cómo la prevención de conductas de autoexterminación de los usuarios de la APS es llevada a cabo por el equipo interdisciplinario del servicio de salud. Para ello, se realizó una revisión sistemática, siguiendo las directrices PRISMA, con base en estudios brasileños publicados, en formato de artículo científico original, en la base de datos SciELO y LILACS, entre los años 2016 y 2021. Se recuperaron 28 artículos, de los cuales 7 fueron seleccionados según los criterios de inclusión, para componer la muestra. Se encontró que si bien la APS cuenta con estrategias para actuar de manera preventiva en el manejo de conductas orientadas al auto-exterminio, entre estas, la facilidad para crear vínculos, la atención al paciente, la identificación, valoración y seguimiento regular del individuo y de los factores de riesgo, de factores desencadenantes de protección y visitas domiciliarias, los profesionales aún se muestran incapaces e indefensos para ejercer la gestión de sus usuarios. En este sentido, sugiere nuevos estudios sobre el tema, que involucren propuestas y soluciones para remediar las dificultades encontradas por estos profesionales, así como una adecuada formación y el desarrollo de estrategias para prevenir este fenómeno.

Palabras clave: Suicidio; Atención primaria de salud; Estrategia de salud de la familia.

\section{Introdução}

O comportamento suicida é caracterizado por toda ação na qual o ser humano provoca lesão a si próprio, independente da sua intenção quanto à letalidade ou do motivo que está relacionado ao ato (Brito et al., 2020). Tal comportamento envolve a ideação, o planejamento e as tentativas; ou seja, engloba os pensamentos sobre autoaniquilação, o desenvolvimento de estratégias especificas para atingir o objetivo e por fim, o próprio ato, que poderá resultar em um desfecho fatal (Claumann et al., 2017).

Em todo o mundo, estima-se que a cada quarenta segundos, uma pessoa é vítima de suicídio, o que é responsável por aproximadamente 800 mil mortes anualmente. O suicídio acomete principalmente indivíduos do sexo masculino e com idade igual ou superior aos 70 anos. Além disso, está associado à segunda principal causa de morte entre jovens de 15 a 29 anos, totalizando 8,5\% dos óbitos nessa faixa etária durante o ano de 2012 (Opas, 2014).

O Brasil ocupa a oitava posição no mundo em número de suicídios. Nesse cenário, constata-se que o risco de suicídio no sexo masculino é cerca de quatro vezes maior quando comparado ao sexo feminino, acometendo, também, principalmente indivíduos com mais de 70 anos de idade. Em contrapartida, nota-se que os índices relacionados ao planejamento e a tentativas de suicídios são maiores na população feminina (Brasil, 2017; Ribeiro et al., 2018).

De modo geral, observa-se que as maiores taxas de suicídio no país estão vinculadas à população de solteiros, de viúvos e de divorciados, em ambos os sexos. Os índices de suicídio também são alarmantes em populações que frequentemente são vítimas de discriminação, como em (i) indígenas, (ii) migrantes, (iii) refugiados, (iv) pessoas privadas de liberdade e (v) pessoas do grupo das minorias sexuais, como lésbicas, gays, bissexuais, transgêneros e intersexuais (Brasil, 2017).

Além do próprio ato, a ideação suicida representa um grande indício de ameaças e problemas para a saúde, visto que, segundo o Boletim epidemiológico do Ministério da Saúde, sobre suicídio, para cada indivíduo que comete suicídio, existem aproximadamente outras vinte pessoas que tentaram a ação. Nesse sentido, a história pregressa de tentativa suicida torna-se o principal fator de risco para a atitude (Brasil, 2017; Sousa et al., 2020). 
O comportamento de dar fim à própria existência configura um fenômeno complexo e multifatorial, que irá ocorrer em diferentes contextos, sendo a consequência final da interação de diversos fatores sociais, culturais, psicológicos, biológicos e ambientais. Dessa forma, tal prática, não pode ser explicada por nenhum fator isolado, necessitando, então, de um cuidado específico e longitudinal de cada indivíduo (WHO, 2014; Ribeiro et al., 2018).

A compreensão quanto à sua incidência atual, sobre as peculiaridades de cada indivíduo afetado, assim como das características em comum dessas pessoas e o conhecimento dos locais que mais são acometidos, possibilitam a elaboração de ações preventivas locais, desenvolvidas tanto pelos serviços de saúde quanto pela própria comunidade, que irão contribuir na identificação da população de risco e consequentemente na diminuição do número de vítimas (Franck; Monteiro; Limberger, 2020).

Levando em consideração que a Atenção Primária à Saúde (APS) é a porta de entrada preferencial dos usuários nos serviços de saúde, somado ao fato de que os profissionais que fazem parte dela são capazes de oferecer um cuidado continuado ao indivíduo, além de conhecer as particularidades da sua clientela adscrita, tal contexto, torna-se um ambiente propício para a assimilação de características que possam auxiliar no reconhecimento da ideação suicida. Nessa perspectiva, a APS passa a exercer um papel crucial na identificação, na atenção, no manejo e em ações de prevenção e posvenção - esse último conceito consiste em cuidado dedicado aos enlutados por uma circunstância de autoextermínio - do comportamento suicida (OMS, 2000; Padilha et al., 2019).

Dessa forma, destaca-se a posição estratégica que a Atenção Primária a Saúde assume em comparação aos demais serviços de saúde, uma vez que está intimamente inserida no território, possuindo facilidade em seu acesso e construindo um vínculo com seus pacientes; fatores que contribuem para o manejo adequado de seus usuários, bem como a elaboração de estratégias de prevenção que atenda às verdadeiras necessidades da população, visando a redução dos índices de suicídio (Ferreira et al., 2018). Diante do exposto, o presente estudo objetivou revisar, na literatura científica, como se dá o manejo na prevenção do comportamento suicida dos usuários da Atenção Primária à Saúde pela equipe interdisciplinar do serviço de saúde.

\section{Metodologia}

Trata-se de uma revisão bibliográfica sistemática de literatura de natureza qualitativa, seguindo as diretrizes PRISMA, através de busca por estudos brasileiros publicados, no formato de artigo científico original, entre os anos de 2016 e 2021 . A revisão sistemática é um método de investigação cientifica, caracterizada pelo objetivo de agrupar, analisar de forma crítica e elaborar uma síntese à partir dos resultados obtidos através de diversos estudos primários (Cordeiro, et al., 2007). Segundo Pereira et al (2018), métodos qualitativos são aqueles nos quais a interpretação e opiniões sobre o fenômeno em estudo, por parte do pesquisador, são fundamentais.

Como critérios de inclusão, foi realizada uma pesquisa por artigos que abordam as seguintes temáticas: i) Comportamento suicida; ii) O papel da Atenção Primária à Saúde diante o comportamento suicida dos usuários iii) Manejo na prevenção do comportamento suicida dos usuários da Atenção Primária à Saúde, publicados em língua portuguesa, feitos no Brasil e disponíveis na forma de texto completo.

Em relação aos critérios de exclusão, foram excluídos: I) artigos de revisão, teses, dissertações, resumos em anais de eventos e estudos de textos indisponíveis; II) artigos dentro da temática, porém não escritos em língua portuguesa; III) artigos dentro da temática, porém não realizados no Brasil; IV) artigos dentro da temática, porém publicados antes de 2016; V) artigos que tratam do comportamento de autoextermínio, não relacionado a APS; e VI) artigos sobre APS sem abordagem da temática autoextermínio. 
A pesquisa foi realizada no período de maio de 2021 a junho de 2021, nas bases de dados on-line Scientific Electronic Library Online (SciELO) e Literatura Latino Americana e do Caribe em Ciências Sociais e da Saúde (LILACS). Foram utilizados os descritores em ciências da saúde (DeCS) associados ao operador booleano AND, da seguinte maneira: "Suicídio AND Integralidade em Saúde”, "Suicídio AND Atenção Primária à Saúde”, "Suicídio AND Estratégia Saúde da Família”.

Na base de dados SciELO (https://www.scielo.org/), foram utilizados os filtros "Brasil", de publicações a partir de 2016, até junho de 2021, no idioma "português" e "artigo" e, na base de dados LILACS (https://lilacs.bvsalud.org/), foram utilizados os filtros "português" "texto completo" de publicações do ano de 2016 até junho de 2021.

Baseando-se nos critérios de inclusão e de exclusão pré-estabelecidos, foi realizada a seleção dos artigos a partir da leitura de: (i) título; (ii) resumo; e (iii) palavras-chaves. Os resultados foram expostos após leitura e interpretação dos textos completos com compilação dos dados e informações obtidas, a partir da utilização de fluxograma PRISMA.

Os resultados são apresentados da seguinte forma: (i) Características do comportamento suicida, (ii) Faixa etária mais acometida por ocorrências de suicídio, (iii) Profissionais de saúde e identificação de comportamento suicida: como conduzir o caso? (iv) Papel da equipe multiprofissional e interdisciplinar na abordagem terapêutica de pacientes que tentaram ou que são propensos a tentar autoextermínio (v) APS, diagnóstico e manejo do paciente com comportamento suicida.

\section{Resultados e Discussão}

Utilizando os descritores "Suicídio AND Integralidade em Saúde", "Suicídio AND Atenção Primária à Saúde" e "Suicídio AND Estratégia Saúde da Família", nas bases de dados SciELO e LILACS, foram recuperados um total de 28 publicações, conforme mostra o Quadro 1.

Quadro 1 - Quantitativo de artigos obtidos em cada base de dados científicos, de acordo com o descritor utilizado.

\begin{tabular}{|l|c|c|}
\hline \multicolumn{1}{|c|}{ DESCRITOR } & SciELO & LILACS \\
\hline "Suicídio AND Integralidade em Saúde" & 2 & 1 \\
\hline "Suicídio AND Atenção Primária à Saúde" & 4 & 14 \\
\hline "Suicídio AND Estratégia Saúde da Família" & 2 & 5 \\
\hline
\end{tabular}

Fonte: Autores (2021).

$\mathrm{Na}$ base de dados SciELO foram recuperados 8 artigos, enquanto na base de dados LILACS obteve-se 20 artigos, com a utilização dos filtros. Após a aplicação dos critérios de inclusão e exclusão, restaram 7 artigos que foram incluídos neste estudo, conforme está descrito no fluxograma Prisma da Figura 1. 
Figura 1 - Fluxograma Prisma.

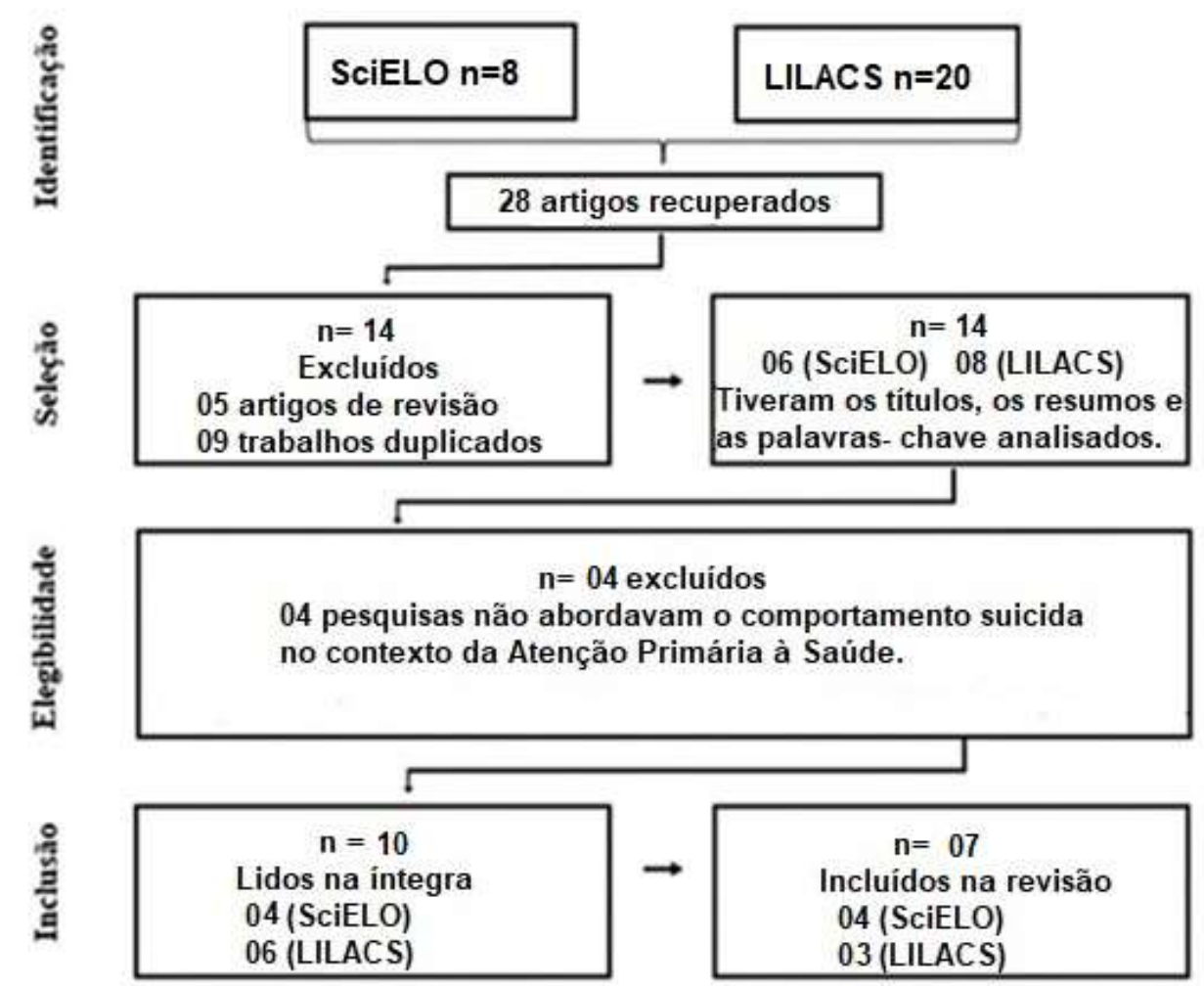

Fonte: Autores (2021).

Esses artigos foram analisados, na integra, a fim de interpretá-los e discuti-los. Quanto as características gerais, os 7 artigos que constituem a amostra, estão inseridos no contexto da Atenção Primária à Saúde, sendo que a publicação mais antiga era do ano de 2017. Quanto a abordagem do estudo, 5 eram estudos qualitativos e os outros 2 quantitativos. Os achados estão apresentados no Quadro 2.

Quadro 2 - Artigos incluídos na pesquisa contemplando os seguintes aspectos: Título, autor, ano, objetivo e principais resultados.

\begin{tabular}{|c|c|c|c|}
\hline TÍTULO & AUTOR E ANO & OBJETIVO & PRINCIPAIS RESULTADOS \\
\hline $\begin{array}{l}\text { Possibilidades de abordagem do } \\
\text { tema do suicídio na Estratégia } \\
\text { Saúde da Família. }\end{array}$ & $\begin{array}{l}\text { Ferreira; Fajardo; } \\
\text { Mello; } 2019 .\end{array}$ & $\begin{array}{l}\text { Conhecer como profissionais de } \\
\text { uma unidade de Estratégia Saúde } \\
\text { da Família (ESF) lidam com o } \\
\text { comportamento suicida }\end{array}$ & $\begin{array}{l}\text { Foram identificadas as dificuldades que } \\
\text { ocorrem no trabalho como comportamento } \\
\text { suicida assim como os efeitos psíquicos que } \\
\text { provocam nos profissionais. }\end{array}$ \\
\hline $\begin{array}{l}\text { Pesquisa-ação sobre saberes e } \\
\text { práticas de agentes comunitários } \\
\text { de saúde acerca da prevenção do } \\
\text { comportamento suicida. }\end{array}$ & Junior et al., 2021 & $\begin{array}{l}\text { Analisar práticas e saberes dos } \\
\text { agentes comunitários de saúde } \\
\text { (ACS) à respeito da prevenção do } \\
\text { comportamento suicida. }\end{array}$ & $\begin{array}{l}\text { Os saberes dos ACS sobre comportamento } \\
\text { suicida envolvem fatores desencadeadores, } \\
\text { como traumas emocionais. A identificação } \\
\text { de sinais de alerta, monitoramento da pessoa } \\
\text { em situação de risco e orientações fazem } \\
\text { parte da prevenção ao suicídio. }\end{array}$ \\
\hline $\begin{array}{l}\text { Educação permanente em } \\
\text { saúde mental: o suicídio na } \\
\text { agenda do cuidado dos } \\
\text { Agentes Comunitários de } \\
\text { Saúde }\end{array}$ & $\begin{array}{l}\text { Medeiros; } \\
\text { Medeiros; Pinto; } \\
2020 .\end{array}$ & $\begin{array}{l}\text { Avaliar as atitudes dos ACS } \\
\text { frente ao comportamento suicida } \\
\text { antes e depois da capacitação } \\
\text { voltada para o manejo de casos } \\
\text { com risco suicida. }\end{array}$ & $\begin{array}{l}\text { Os resultados indicaram que depois da } \\
\text { capacitação os ACS apresentaram atitudes } \\
\text { mais positivas sobre a capacidade para lidar } \\
\text { com os pacientes. }\end{array}$ \\
\hline
\end{tabular}




\begin{tabular}{|l|l|l|l|}
\hline $\begin{array}{l}\text { Assistência de enfermagem } \\
\text { na atenção primária à saúde } \\
\text { de adolescentes com ideações } \\
\text { suicidas }\end{array}$ & $\begin{array}{l}\text { Pessoa et al., } \\
2020 .\end{array}$ & $\begin{array}{l}\text { Compreender a assistência à } \\
\text { saúde prestada pelos enfermeiros } \\
\text { na atenção primária aos } \\
\text { adolescentes com comportamento } \\
\text { suicidas. }\end{array}$ & $\begin{array}{l}\text { Evidencia-se a falta de planejamento e de } \\
\text { ações no âmbito da saúde mental dos } \\
\text { adolescentes. Os enfermeiros apresentam } \\
\text { dificuldades em identificar e prevenir os } \\
\text { sinais de ideações suicidas, pautando sua } \\
\text { prática em experiências. }\end{array}$ \\
\hline $\begin{array}{l}\text { Atitudes dos profissionais no } \\
\text { cuidado em situação de } \\
\text { suicídio: estudo transversal }\end{array}$ & $\begin{array}{l}\text { Silva \& Souza, } \\
\text { 2021. }\end{array}$ & $\begin{array}{l}\text { Conhecer as crenças e atitudes } \\
\text { dos profissionais da ESF de Santa } \\
\text { Cruz Cabrália/Bahia sobre } \\
\text { suicídio. }\end{array}$ & $\begin{array}{l}\text { Evidencia-se um despreparo dos } \\
\text { profissionais, além de sentimentos de } \\
\text { incapacidade e atitudes moralistas. no } \\
\text { atendimento a usuários em risco de suicídio. }\end{array}$ \\
\hline $\begin{array}{l}\text { Ações do enfermeiro na } \\
\text { atenção básica para } \\
\text { prevenção do suicídio }\end{array}$ & Silva et al., 2017. & $\begin{array}{l}\text { Descrever as ações realizadas } \\
\text { pelo enfermeiro da atenção básica } \\
\text { para prevenção do suicídio. }\end{array}$ & $\begin{array}{l}\text { Revelou que as ações para prevenção do } \\
\text { suicídio na atenção básica necessitam ser } \\
\text { inseridas no processo de trabalho desses } \\
\text { profissionais. }\end{array}$ \\
\hline $\begin{array}{l}\text { Prevenção ao suicídio na } \\
\text { atenção básica: concepção de } \\
\text { enfermeiros }\end{array}$ & Sousa et al., 2019. & $\begin{array}{l}\text { Descrever a opinião de } \\
\text { Enfermeiros da Atenção Básica } \\
\text { acerca da prevenção do suicídio. }\end{array}$ & $\begin{array}{l}\text { Evidenciou-se a carência de capacitação } \\
\text { como uma lacuna na atuação de Enfermeiros } \\
\text { na prevenção do suicídio. }\end{array}$ \\
\hline
\end{tabular}

Fonte: Autores (2021).

Para a apresentação dos resultados compilados, optou-se por descrever os mesmos nos itens expostos na sequência: (i) Características do comportamento suicida, (ii) Faixa etária mais acometida por ocorrências de suicídio, (iii) Profissionais de saúde e identificação de comportamento suicida: como conduzir o caso? (iv) Papel da equipe multiprofissional e interdisciplinar na abordagem terapêutica de pacientes que tentaram ou que são propensos a tentar autoextermínio (v) APS, diagnóstico e manejo do paciente com comportamento suicida.

\subsection{Características do comportamento suicida}

O comportamento suicida é um tema enigmático, ainda condenado pela sociedade, devido à sua complexidade e à obscuridade que envolve o ato, uma vez que este contrapõe o instinto primordial de sobrevivência do ser humano. Entender os motivos que levam um indivíduo a idealizar e planejar a sua própria morte, assim como escolher o próprio método para executar a ação é difícil, no entanto, acredita-se que a vontade de aliviar um sofrimento emocional coloca o sujeito frente às diversas formas do comportamento de autoextermínio (Marçal \& Gonçalves, 2020).

Os limites entre a ideação suicida, a tentativa de tirar a própria vida e a ação propriamente dita é muito sútil. Geralmente, o sofrimento e as tristezas que fomentam a ideia ou intenção de autoagressão podem atingir um nível extremo e levar ao ato. Esse discreto limite alerta que o suicídio é, quase sempre, idealizado, planejado e precedido de tentativas, sendo possível então, a intervenção por meio de estratégias de prevenção. Porém, tal fato, não descarta situações que possam ocorrer por impulso desesperador e que não foram planejados previamente (Teixeira; Souza; Viana, 2018).

Destaca-se que o indivíduo que apresenta ideação suicida poderá evidenciar sinais de alertas verbais como, por exemplo, comentários em que manifesta o desejo de morrer ou que expressa o fato de não suportar mais viver, além de, inúmeras vezes, considerar-se como um peso na vida dos outros. Somado a isso, esses pacientes podem, também, apresentar alterações comportamentais, manifestando sofrimento intenso, autoestima baixa, alterações repentinas do humor, isolamento social, ausência de esperança, impulsividade e, até mesmo, podem elaborar preparativos para a sua própria morte, seja escrevendo bilhetes destinados a pessoas do seu convívio social ou presenteando, como forma de recordação, amigos e familiares com objetos pessoais que possuem valor sentimental (Lima et al., 2018).

De modo geral, observa-se que esse fenômeno está associado a fatores de riscos e também a fatores considerados como protetores. A presença de determinados fatores de riscos pode resultar no desenvolvimento do comportamento de autoagressão com intuito de dar fim à sua própria vida. Dentre eles, podemos citar fatores sociais como situações estressoras 
que envolvam a perda de figuras parentais na infância ou recentemente, estrutura familiar conturbada ou personalidade com características de agressividade e/ou impulsividade (Almeida et al., 2020).

Os estudos apontam a importância do conhecimento dos índices de ocorrência do suicídio, assim como das características biopsicossociais envolvidas no fenômeno, uma vez que, fatores de riscos precisam ser identificados e posteriormente acompanhados através de programas de prevenção e tratamento eficaz, resultando em uma diminuição dos eventos suicidas (Ferreira et al., 2014).

Em relação ao perfil das vítimas, destaca-se a vulnerabilidade feminina. Mulheres tendem a tentarem suicídio mais vezes quando comparado aos homens, porém, o sexo masculino tem mais sucesso no ato. Tal fato está relacionado aos métodos do suicídio, uma vez que homens optam por estratégias mais letais como armas, enforcamento, enquanto, as mulheres utilizam das diversas formas de envenenamento (Tadiello \& Seixas, 2020).

Além disso, há evidências de uma forte ligação entre os transtornos mentais e o comportamento suicida, sendo que inúmeros indivíduos que cometeram suicídio possuíam um transtorno mental diagnosticável, assim como, em pacientes psiquiátricos, esse fenômeno é uma manifestação comum. Entre os principais transtornos mentais associados ao suicídio, destaca-se o transtorno depressivo maior, estando presente em cerca de 40 a $50 \%$ da população que tentou autoextermínio, a esquizofrenia presente em $10 \%$ dos casos e o transtorno por uso de álcool, presente em aproximadamente $20 \%$ das vítimas (Ferreiraet al., 2018; Aguiar; Azevedo; Ferretjans, 2015).

Ao contrário dos fatores de risco, os elementos considerados de proteção podem atenuar as consequências dos eventos negativos e das contrariedades enfrentadas pelo ser humano durante a sua vida. Alguns fatores de proteção são as características pessoais, como a autoestima do indivíduo, e as características do meio em que se encontra inserido como, por exemplo, a relação com a família, amigos e a rede de apoio, responsáveis por fornecerem suporte em diversas situações (Pereira et al., 2018).

Nesse sentido, o comportamento suicida não é caracterizado como uma patologia, mas sim, um conjunto de diferentes fatores que impulsionam um indivíduo a desenvolvê-lo, em grande parte dos casos, associados a outras patologias clinicas ou a transtornos psiquiátricos. Portanto, a incompreensão dos seus desencadeadores, assim como o desconhecimento do próprio ato e da sua prevenção, é responsável pelo aumento da mortalidade em todo mundo por lesões autoprovocadas (Ferreira, 2020).

No entanto, apesar dos estudos evidenciarem associações significativas que possam potencializar o risco suicida, nenhum desses fatores de risco separadamente ou em conjunto com outros fatores irão determinar com precisão aqueles indivíduos que irão ou não tentar autoextermínio. Dessa forma, o julgamento clinico baseado na história completa do paciente e na análise minuciosa das particularidades de cada indivíduo - como a etapa de vida - é fundamental para determinação de potenciais vítimas (Del-Ben et al., 2017).

\subsection{Faixa etária mais acometida por ocorrências de suicídio}

No que diz respeito às etapas da vida, estudos mostram a adolescência como a fase com maior índice de suicídio, sendo que nesse período marcado por crises profundas decorrentes das transformações físicas, psicológicas e culturais, aproximadamente um a cada oito jovens tentam se suicidar. Já em relação aos idosos, considerando o envelhecimento populacional, o fenômeno merece destaque, uma vez que as taxas cresceram muito nos últimos anos, nessa população, e esses indivíduos tendem a comunicar menos suas ideações bem como a adotarem métodos mais letais (Schlösser; Rosa; More, 2014).

Nesse sentido, verifica-se que o suicídio é um grande problema de saúde pública, representando as principais causas de morte no cenário mundial, dessa forma, o seu aumento progressivo a cada ano tem sido motivo de estudos e discussões na literatura. No entanto, acredita-se que esses números sejam subestimados, mascarando as verdadeiras taxas desse fenômeno e 
gerando uma análise irreal da situação na população (Silva \& Marcolan, 2019).

Nota-se que, apesar dos grandes municípios apresentarem uma disponibilidade maior de médicos que investigam mortes violentas, altas taxas dessas mortes ainda se mantem indeterminadas. Em contrapartida, nos municípios pequenos, um episódio como o suicídio é incomum passar despercebido. Dessa forma, parte de taxas baixas de mortalidade por esse fenômeno em municípios maiores pode estar relacionado à subnotificação (Silva; Junior; Suchara, 2018).

Outros fatores que podem estar relacionados a essa subnotificação são o preenchimento incorreto da certidão de óbito, cemitérios clandestinos e pedidos oriundos da família para que se altere o motivo da morte. Observa-se também uma ausência de fluxos para notificação dos atendimentos, nas ocorrências de tentativas de suicídio em serviços de urgência e emergência, o que dificulta ações de prevenção e de condução de autoextermínio proporcionadas por profissionais de saúde, quando do diagnóstico da situação de risco (Silva \& Marcolan, 2019).

\subsection{Profissionais de saúde e identificação de comportamento suicida: como conduzir o caso?}

O comportamento suicida pode atingir o ser humano em diferentes períodos da vida e consequentemente desencadear impactos no âmbito familiar, nos ciclos de amizades e até mesmo na comunidade, de modo que, torna-se necessário auxílio nessas diferentes esferas para enfrentar as angústias geradas por esse fenômeno. Dessa forma, a ação dos profissionais de saúde engloba desde o reconhecimento precoce da ideia de autoaniquilação, o acolhimento diante os episódios de crise, assim como o apoio direto ou indiretamente a vítima e seu círculo social (Ferreira, 2020).

No primeiro momento, a avaliação de risco suicida tem como finalidade identificar a existência de ideação e planejamento suicida, além de analisar a gravidade e a letalidade de tentativas prévias e das atuais. Somado a isso, é necessário identificar os fatores de risco e os elementos protetores, verificar a existência de diagnóstico psiquiátrico prévio, assim como instituir terapêutica inicial para as condições de base. Por fim, é necessário garantir a inserção do paciente em serviços de saúde mental. Ressalta-se que essa avaliação deve ser conduzida com privacidade, em ambiente tranquilo e a abordagem deve ser clara e acolhedora (Del-Ben et al., 2017).

Após a identificação do risco de suicídio, dialogando com Aguiar, Azevedo e Ferretjans (2015), o estudo de Júnior e colaboradores (2021), aponta que é fundamental investir nos métodos de proteção, como por exemplo, práticas de esportes, momentos de lazer, espiritualidade e até mesmo acionar a família do paciente para que essa participe do seu cuidado, visto que a maior parte das vítimas comunicam abertamente essa intenção aos seus familiares. Segundo os autores, a família exerce um papel essencial no trabalho de evitar que o indivíduo cometa suicídio e deve ser orientada a não deixar o indivíduo sozinho, bem como evitar que ele tenha acesso a objetos como medicamentos, facas, armas, fogo, instrumentos que possam ser utilizados para a execução do ato.

Outro ponto importante é a notificação compulsória e imediata, tanto de casos suspeitos, como de presença confirmada do ato, à vigilância epidemiológica do município pelo profissional de saúde, em até 24 horas após o atendimento, assim como realizar a comunicação ao Conselho Tutelar quando envolver crianças e adolescentes, e ao Conselho do Idoso ou ao Ministério Público, em casos de pessoas que possuem 60 anos de idade ou mais. Tal estratégia é importante para gerar informações a respeito da frequência de suicídio e, consequentemente, estabelecer medidas de proteção eficazes a esses indivíduos bem como, favorecer a criação de políticas públicas e estratégias para prevenção do suicídio (Brasil, 2017).

Ademais, reconhece-se que cada caso exige uma intervenção específica, pelos profissionais de saúde, de modo a compreender o significado que o comportamento tem na vida daquela vítima, uma vez que diferentes indivíduos podem apresentar reações distintas, diante de contextos semelhantes, de forma que, situações de estresse para determinadas pessoas podem acarretar ao ato de autoextermínio enquanto para outros não, o que caracteriza o suicídio como um fenômeno complexo (Machado, 2011). 


\subsection{Papel da equipe multiprofissional e interdisciplinar na abordagem terapêutica de pacientes que tentaram ou que são propensos a tentar autoextermínio}

$\mathrm{Na}$ área da saúde, especialmente no universo da saúde mental, o vínculo é a consequência do encontro entre o profissional e o paciente, sendo este, uma das formas de cuidado mais potente e eficaz no que se diz respeito à promoção da saúde. O estabelecimento de vínculos possibilita entender profundamente sobre o processo de saúde e doença, assim como compreender as verdadeiras necessidades de saúde do ser humano (Oliveira et al., 2017).

Partindo desse pressuposto, o estudo de Pessoa et al. (2020) evidencia que um bom acolhimento possibilita a criação de vínculos e, consequentemente, permite o desenvolvimento de estratégias para prevenção e promoção a saúde. Ferreira, Fajardo e Mello (2019) acrescenta ainda, que inúmeras vezes, existe a falsa ideia de que o profissional precisa fazer ou falar algo durante a abordagem do indivíduo com risco suicida, sendo que, por muitas vezes, a escuta de forma acolhedora é a terapêutica que muitos pacientes precisam naquele momento.

Nessa perspectiva, a prática de solidariedade, mediante estratégias como o acolhimento e a criação de vínculos por parte dos profissionais de saúde, frente aos indivíduos que tentaram ou são propensos a tentar autoextermínio são fundamentais para identificação de potenciais vítimas e consequentemente redução desses trágicos índices. Dessa forma, pacientes com características que demonstram sentimentos como angústia, desesperança, desamparo, solidão e desespero devem ser abordados de forma clara, cautelosa e empática pela equipe multidisciplinar de saúde (Marçal \& Gonçalves, 2020).

O estudo de Junior et al. (2021), realizado com 13 agentes comunitários de saúde (ACS) corrobora com a ideia apresentada acima, e afirma que diante a identificação desses sentimentos, os pacientes devem ser avaliados e acompanhados regularmente, a fim de que desfechos negativos possam ser evitados. Ademais, ressalta a importância que os ACS têm frente ao comportamento suicida, principalmente por viverem no local onde trabalham, sendo capazes de conhecer o território, além de ser o elo entre a comunidade e o serviço de saúde.

Além disso, Sousa et al. (2019) acrescenta que a visita domiciliar é uma das inúmeras alternativas que a Atenção Primária à Saúde apresente para a elaboração de atividades preventivas frente ao comportamento suicida, uma vez que, essa estratégia permite o levantamento de casos possíveis para o suicídio e consequente monitoramento das potenciais vítimas.

Uma situação que pode ser obstáculo diante às ações de prevenção do suicídio pelas equipes de saúde são os rótulos e preconceitos que o comportamento de autoextermínio carrega. Vítimas desse fenômeno estão sujeitas a enfrentar julgamentos negativos por toda a comunidade, o que pode dificultar o processo de busca por ajuda e culminar com um desfecho fatal, sendo então, fundamental para os pesquisadores Sousa et al. (2019) e Storino et al. (2018) que as equipes atuem desmascarando os diversos mitos que o suicídio carrega, para que esses sujeitos não se sintam envergonhados, rejeitados ou até mesmo discriminados.

Uma das maiores crenças apontadas nos estudos é acreditar que os sujeitos que falam ou que demonstram indícios não irão cometer suicídio e que adotam esse comportamento apenas para chamar atenção, sendo que na realidade, a maior parte dos indivíduos que cometem suicídio expressaram suas ideias por dias ou semanas que precederam o ato com pessoas do seu convívio social ou a profissionais de saúde. Outro mito é pensar que não se pode falar sobre suicídio, acreditando que isso pode aumentar o risco, porém, o silêncio perpetua que o suicídio seja um tabu, comprometendo que o indivíduo alivie suas tristezas e angustias. Por último, há a falácia de que sujeitos que tentam suicídio possuem risco por toda sua vida, sendo que, se o tratamento for efetivo, o paciente não possui o mesmo risco de antes (Almeida et al., 2020).

No entanto, de acordo com Silva e Souza (2021), nota-se que apesar da necessidade de desmistificar o comportamento de autoextermínio, as atitudes dos profissionais ainda são cercadas dessas crenças e estigmas, o que pode levar a uma assistência inadequada. Segundo os autores, uma equipe despreparada pode levar ao agravamento da condição do paciente, uma vez que este pode se sentir desconfortável e consequentemente afastar-se do serviço, evitando que esses indivíduos 
busquem ajuda em uma próxima ocasião.

Nesse sentido, compreende-se que a prevenção do comportamento suicida não é um trabalho simples. Dessa forma, para que se faça possível a identificação precoce de indivíduos com ideias suicidas bem como a intervenção precoce e manejo adequado desses indivíduos, a fim de reduzir o risco de potenciais vítimas, os estudos de Silva et al. (2017) e Silva e Souza (2021) concordam com a ideia de que é imprescindível que, principalmente na Atenção Básica, os profissionais estejam bem capacitados e estruturados para a promoção da resolutividade em situações de tentativas de autoextermínio.

\subsection{APS, diagnóstico e manejo do paciente com comportamento suicida}

O Sistema de Atenção à Saúde (SAS) brasileiro é estruturado em Redes de Atenção à Saúde (RAS), caracterizadas como um sistema integrado que articula de forma horizontal, em todas as esferas de atenção, os diversos níveis de complexidade, ou seja, na atenção primária, secundária e terciária. Dessa forma, tem como propósito, garantir a integralidade do cuidado do ser humano, considerando a pluralidade das necessidades de saúde da população brasileira (Sousa et al., 2019).

Diante o comportamento suicida, os serviços de saúde amparam os indivíduos vítimas desse fenômeno, através de dispositivos que se encontram articulados nos diferentes pontos de atenção, de forma que ofereça resolutividade para a sua situação. Entre os dispositivos da rede de atenção à saúde que serão acionados, podemos apontar a atenção hospitalar, a atenção psicossocial estratégica e a atenção básica em saúde, configurando pontos de atenção terciária, secundária e primária, respectivamente (Stoppa; Wanderbroocke; Azevêdo, 2020).

Observa-se que, em cada cenário e em diferentes ocasiões, há uma equipe multidisciplinar que exerce a assistência aos pacientes que tentaram autoextermínio; no entanto, mesmo considerando as peculiaridades na organização dos serviços de saúde, evidencia o papel que a Atenção Primária à Saúde tem papel relevante na identificação e na prevenção do comportamento suicida, assim como no reconhecimento dos aspectos biopsicossociais relacionados a esse processo, que são consequentemente impulsionadores de toda angústia vivenciada (Ferreira et al., 2018).

Estudos apontam que a grande maioria dos indivíduos que cometem o ato de autoaniquilação, ao decorrer da sua vida, nunca consultou com um profissional de saúde mental, entretanto, sabe-se que cerca de metade desses pacientes compareceram a uma consulta médica, no intervalo de seis meses que precederam a morte, sendo que, dentre esses, a maior parte dos pacientes, foi atendida por um médico generalista, no mês anterior ao episódio (Aguiar; Azevedo; Ferretjans, 2015).

Nessa perspectiva, os estudos de Ferreira, Fajardo e Mello (2019) e de Sousa et al. (2019) corroboram com a ideia de que considerar a prevenção do comportamento suicida, no domínio da saúde pública, requer analisar a respeito da atuação da APS frente a esse problema. Por ser a porta de entrada preferencial dos usuários ao sistema de saúde, representando o primeiro contato que o usuário tem com o serviço de saúde, além de coordenadora do cuidado, no Sistema Único de Saúde, destaca-se a importância da atuação dos profissionais de saúde neste ponto da rede para a prevenção do fenômeno.

$\mathrm{Na}$ Atenção Primária à Saúde, os profissionais atuam em um território, área geograficamente conhecida, possibilitando proximidade com a comunidade, além do conhecimento da história de vida dos indivíduos e consequentemente estabelecendo um vínculo com seus usuários. Partindo desse pressuposto, o cuidado nesse ponto de atenção é ratificado pela sua profunda inserção no território, bem como a facilidade de acesso das equipes aos usuários e vice-versa (Ferreira, 2020).

Dessa forma, os profissionais da atenção básica à saúde têm a função de reconhecer, avaliar os pacientes com risco de suicídio, realizar os encaminhamentos necessários para a rede de saúde mental, além de acompanhar o usuário na rede de atenção à saúde, promovendo um cuidado longitudinal e integral desse indivíduo. Sendo assim, destaca-se que, para o manejo adequado, é importante que ocorra a articulação entre a atenção básica e os outros pontos da rede, além do preparo dos profissionais para lidar com a situação (Stoppa; Wanderbroocke; Azevêdo, 2020).

No entanto, Sousa et al. (2019) demonstra que embora a atenção básica seja composta por uma equipe 
multidisciplinar, capaz de solucionar e lidar frente à indivíduos com comportamento suicida, uma das primeiras condutas para esses pacientes é o encaminhamento para os serviços especializados, tal atitude, reflete diretamente em uma sobrecarga desses serviços e dos profissionais. Pessoa e colaboradores (2020), também contribui com a ideia de que, idealmente apenas os casos mais complexos deveriam ser encaminhados e que as estratégias de prevenção devem e podem acontecer na Atenção Primária à Saúde.

Nesse sentido, Silva et al. (2017) complementa que apesar dos profissionais compreenderem sobre a necessidade de planejamento de ações preventivas, bem como a identificação de fatores de risco para o suicídio e de que a atuação das equipes nesses fatores, podem ser eficazes no desfecho do comportamento suicida, ainda não existem ações organizadas práticas que possibilitem a identificação de risco e vulnerabilidade relacionadas ao suicídio. Durante a pesquisa realizada, outros estudos também corroboram com essa ideia e apontam entraves presentes no manejo do comportamento suicida. Essas dificuldades estão melhor apresentadas no Quadro 3.

Quadro 3 - Dificuldades apontadas por categorias profissionais no manejo da prevenção do comportamento suicida na Atenção Primária à Saúde.

\begin{tabular}{|c|c|c|}
\hline $\begin{array}{l}\text { Fonte (Autor } \\
\text { e ano) }\end{array}$ & $\begin{array}{l}\text { Profissional } \\
\quad-\text { APS }\end{array}$ & Dificuldades \\
\hline Ferreira; Fajardo; Mello; 2019. & $\begin{array}{c}\text { Médicos } \\
\text { Enfermeiros } \\
\text { Técnicos de enfermagem } \\
\text { ACS }\end{array}$ & $\begin{array}{l}\text { Demanda em excesso, ausência de capacitação, falta de } \\
\text { formação específica sobre o assunto na formação e durante o } \\
\text { trabalho }\end{array}$ \\
\hline Júnior et al., 2021. & ACS & $\begin{array}{c}\text { Dificuldade de abordagem, falta de capacitação dos } \\
\text { profissionais na área de Saúde Mental. Dificuldades em ofertar } \\
\text { cuidado longitudinal. }\end{array}$ \\
\hline Medeiros; Medeiros; Pinto, 2020. & ACS & $\begin{array}{l}\text { Situações precárias de trabalho, falta de motivação, } \\
\text { sobrecarga, falta de suporte técnico científico, adoecimento } \\
\text { relacionado ao trabalho. }\end{array}$ \\
\hline Pessoa et al., 2020. & Enfermeiro & $\begin{array}{l}\text { Despreparo para abordar o tema, falta de capacitações do } \\
\text { município, falta de recursos e infraestrutura, falta de } \\
\text { compreensão sobre os pontos da rede de atenção. }\end{array}$ \\
\hline Silva \& Souza, 2021. & $\begin{array}{l}\text { Enfermeiros } \\
\text { Técnicos de enfermagem } \\
\text { Médicos }\end{array}$ & $\begin{array}{l}\text { Falta de incentivo para a formação e a atualização dos } \\
\text { profissionais da ESF na área da saúde mental. }\end{array}$ \\
\hline Silva et al., 2017. & Enfermeiros & $\begin{array}{l}\text { Inabilidade para enfrentar essa questão, falta de recursos, } \\
\text { ausência de conhecimento específico ligado à saúde mental. }\end{array}$ \\
\hline SOUSA et al., 2019. & Enfermeiro & $\begin{array}{c}\text { Falta de tempo, sobrecarga de trabalho, não capacitação, } \\
\text { falta de habilidade. }\end{array}$ \\
\hline
\end{tabular}

Fonte: Autores (2021).

Segundo Ferreira, Fajardo e Mello (2019) a sensação de incapacidade por parte dos profissionais para lidar com esse fenômeno está associada a falta de conhecimento e capacitação sobre o tema. Nesse sentido, ratificando a necessidade de capacitação dos profissionais da Atenção Primária à Saúde para o manejo na prevenção do comportamento suicida, o estudo de 
Medeiros, Medeiros e Pinto (2020) realizado com 38 Agentes Comunitários de Saúde (ACS), aponta que esses profissionais passaram a apresentar menos insegurança, bem como atitudes mais positivas sobre a maneira de lidar com os indivíduos com risco suicida após uma capacitação breve teórico-prática com duração de 04 horas.

Dessa forma, ressalta-se sobre a necessidade da adoção de ações em educação permanente, tornando-se necessário que os gestores de saúde tenham dimensão do problema, capacitando os profissionais de saúde para que esses estejam aptos a lidar adequadamente com os pacientes, considerando o sujeito em sua integralidade e consequentemente contribuindo para uma abordagem e manejo adequado. Ademais, os estudos sugerem o fortalecimento das redes de saúde, uma vez que o suicídio é um problema que demanda intervenções interdisciplinares e intersetoriais e que existem fragilidades profissionais e dos serviços. (Ferreira; Fajardo e Mello, 2019, Silva et al., 2017; Silva \& Souza, 2021).

\section{Considerações Finais}

Acredita-se que a cada ato ou efeito de pôr fim à própria vida, no mínimo seis outros indivíduos terão a sua vida afetada; essa situação é responsável por um impacto social, psicológico e econômico imensurável no contexto de uma família e/ ou comunidade, o que faz do suicídio um grande problema de saúde pública (Silva \& Marcolan, 2019).

No cenário brasileiro, cerca de $17 \%$ da população já considerou em algum período da vida a tentativa de autoextermínio e aproximadamente $75 \%$ dos indivíduos que já tentaram suicídio procuraram serviços de atenção primária antecedentemente ao ato (Aguiar; Azevedo; Ferretjans, 2015). Sendo assim, por se tratar de um evento multidimensional, mas que é evitável desde que haja uma intervenção adequada, a Atenção Primária à Saúde surge como um cenário essencial no manejo dos indivíduos potencialmente suicidas (Ribeiro et al.,2018).

No entanto, observa-se que apesar da Atenção Primária à Saúde possuir inúmeras estratégias para agir preventivamente no manejo do comportamento voltado ao autoextermínio e que a equipe multiprofissional que atua nesse ponto da rede tem a consciência à respeito da importância da prevenção do suicídio, estes profissionais ainda demonstram-se incapazes e desemparados para exercer o manejo na prevenção do comportamento suicida dos seus usuários. Tal fato, está relacionado à inúmeros fatores, dentre esses, a falta de políticas para prevenção do suicídio, a ausência de capacitação desses profissionais, além de sobrecarga de trabalho e infraestruturas precárias.

Algumas limitações para esse estudo foram a quantidade de dados apresentados. Por fim, sugere novos estudos sobre a temática, que envolva propostas e soluções para sanar as dificuldades encontradas por esses profissionais que atuam na Atenção Primária à Saúde, assim como a capacitação adequada e a elaboração de estratégias para a prevenção desse fenômeno.

\section{Referências}

Aguiar, A. M. A., Azevedo, C. M. \& Ferretjans, R. (2015). Manejo do suicídio pelo médico generalista na atenção primária. Revista Eletrônica Parlatorium, $9(2)$.

Almeida, V. A. S., Costa, F. B., Matos, L. N., Pacheco, L. P., Moreira, M. F. F., Sá, P. M. K., Almeida, T. F. \& Faria, H. M. C. (2020). Prevenção do suicídio: informação, conscientização, identificação e manejo, voltados aos membros da equipe de atenção à saúde primária. Analecta, 6 (3).

Brasil. Ministério da Saúde. Notificação de violência interpessoal/autoprovocada. (2017). Portaria GM/MS $n^{o}$ 1271/2014 e SINAN versão 5.0.https://antigo.saude.gov.br/saude-de-a-z/acidentes-e-violencias/41893-notificacao-de-violencia-interpessoal-autoprovocada-portaria-gm-ms-n-1271-2014e-sinan-versao-5-0

Brasil. Ministério da Saúde. Secretaria de Vigilância em Saúde. Suicídio, saber agir e prevenir. (2017).Boletim Epidemiológico.https://www.gov.br/mdh/ptbr/assuntos/acolha-a-vida/bibliografia/2017025PerfilepidemiologicodastentativaseobitosporsuicidionoBrasilearededeatenaoasade.pdf

Brito, M. D. L. D. S., Júnior, F. J. G. S., Costa A. P. C., Silva, J. C., Gonçalves, A. M. S. \& Monteiro C. F. S. (2020). Comportamento suicida e estratégias de prevenção sob a ótica de professores. Esc Anna Nery, 24(4).

Claumann, G. S., Pinto, A. A., Santos, D. A. \& Pelegrini, S. A. (2017). Prevalência de pensamentos e comportamentos suicidas e associação com a insatisfação corporal em adolescentes. J Bras Psiquiatr, 67 (1), 3-9. 
Cordeiro, A. M., Oliveira, G. M., Rentería, J. M. \& Guimarães, C. A. (2007). Revisão sistemática: uma revisão narrativa. Rev Col Bras Cir., 34(6), 428-431.

Del-Ben, C. M., Junior, A. S., Mantovani, C., Faleiros, M. C. M., Oliveira, G. E. C., Guapo, V. G. \& Marques J. M. A. (2017). Emergências psiquiátricas: manejo de agitação psicomotora e avaliação de risco suicida. Medicina (Ribeirão Preto, Online). 50 (1), 98-112.

Ferreira, G. S., Fajardo, A. P.\& Mello, E. D. (2019). Possibilidades de abordagem do tema do suicídio na Estratégia Saúde da Família.Physis.29 (4).

Ferreira, M. L. (2020). Cuidado à pessoa com comportamento suicida na atenção primária à saúde em um município catarinense. Repositório Institucional da UFSC.

Ferreira, M. L., Vargas, M. A. O., Rodrigues, J., Tretin, D., Brehmer, L. C. F. \& Lino, M. M. (2018). Comportamento suicida e atenção primária à saúde. Enferm. Foco, 9 (4), 50-54.

Ferreira, N. S., Pessoa, V. F., Barros, R. B., Figueiredo, A. E. B. \& Minayo, M. C. S. (2014). Fatores de risco relacionados com suicídios em Palmas (TO), Brasil, 2006-2009, investigados por meio de autópsia psicossocial. Ciênc. saúde coletiva, 19 (01).

Franck, M. C., Monteiro, M. G.\& Limberger, R. P. (2020). Mortalidade por suicídio no Rio Grande do Sul: uma análise transversal dos casos de 2017 e 2018. Epidemiol. Serv. Saúde, 29 (2).

Júnior, F. J. G. S., Silva, K. H., Sales, J. C. S., Costa, A. P. C. \& Monteiro, C. F. S. (2021).Pesquisa-ação sobre saberes e práticas de agentes comunitários de saúde acerca da prevenção do comportamento suicida. Interface (Botucatu), 25.

Lima, K. S. A. L., Pontes, C. F., Lima, W. S. \& Véras, G. C. B. (2018). Prevenção ao suicídio na atenção primária à saúde. Conbracis.

Machado, A. C. (2011). A organização das políticas e gestão da rede de assistência na Atenção Primária à situação de risco para o suicídio. NESCON.

Marçal, S. R. S.\& Gonçalves, J. R. (2020). Estratégias de intervenção do enfermeiro diante do comportamento e tentativa de autoextermínio. Revista JRG de Estudos Acadêmicos. 3(6).

Medeiros, B. G., Medeiros, N. S. B.\& Pinto, T. R. (2020). Educação permanente em saúde mental: o suicídio na agenda do cuidado dos Agentes Comunitários de Saúde. Pesqui. prát. psicossociais, 15(2).

Melo, M. M., Fontes, F. L. L., Silva, R. C., Júnior, J. G. A. L., Santos, J. M. S., Pinho, L. F., Brito, T. S., Soares, J. C., Lopes, M. C. F., Sousa, E. K. B., Santana, E., Araújo, M. A., Matos, D. R., Araujo, C. S., Barbosa, S. S., Oliveira, M. C., Silva, M. J. M., Martins, G. S., Coimbra, G. T., Araújo, C. A. \& Araújo, L. M. (2019). Prevenção ao suicídio na atenção básica: discutir para prevenir. Brazilian Journal of Surgery and Clinical Research - BJSCR, 26 (1),7275.

Oliveira, G. C., Schneider, J. F., Santos, V. B. D., Pinho, L. B., Piloti, D. F. W. \& Lava, E. (2017). Cuidados de enfermagem a pacientes com risco de suicídio. Cienc Cuid Saude, 16 (2).

OMS. (2000). Prevenção ao suicídio: um manual para profissionais da aúde em primária.https://www.who.int/mental_health/prevention/suicide/en/suicideprev_phc_port.pdf

OPAS. (2011). Hope and Healing After Suicide: A practical guide for people who have lost someone to suicide in Ontario. Centre for Addiction and Mental Health. http://www.camh.ca/hopeandhealing

Padilha, C. D. S., Casarin, A. P., Guerreiro, B. C., Oliveira, S. A., Pavão, J., Fotes, C., Mokwa, M. E. \& Marcon, S. (2020). Prevenção ao suicídio na atenção primária em saúde: vivência do PET - saúde interprofissionalidade. Saúde Meio Ambient., 9 (1), 91-92.

Pereira, A.S. et al. (2018). Metodologia da pesquisa científica. $\quad 1^{\mathrm{a}}$ edição. Biblioteca Central da UFSM. https://repositorio.ufsm.br/bitstream/handle/1/15824/Lic_Computacao_Metodologia-Pesquisa-Cientifica.pdf?sequence=1

Pereira, A. S., Willhelm, A. R., Koller, S. H. \& Almeida, R. M. M. (2018). Fatores de risco e proteção para tentativa de suicídio na adultez emergente. Ciência \& Saúde Coletiva, 23(11), 2018.

Pessoa, D. M. S., Freitas, R. J. M., Melo, J. A. L., Barreto, F. A., Melo, K. C. O. \& Dias, E. C. S. (2020). Assistência de enfermagem na Atenção Primária à Saúde de adolescentes com ideações suicidas. Rev Min Enferm, 24.

Ribeiro, N. M., Castro, S. S., Scatena, L. M., Haas, V. J. (2018). Análise da tendência temporal do suicídio e de sistemas de informações em saúde em relação às tentativas de suicídio. Texto \& Contexto-Enferm, 27 (2).

Schlösser, A., Rosa, G. F. C.\& More, C. L. O. O. (2014). Revisão: Comportamento Suicida ao Longo do Ciclo Vital. Temas em Psicologia,22 (1), 133-145.

Silva, D. A.\& Marcolan, J. F. (2019). Epidemiologia do suicídio no Brasil entre 1996 e 2016 e a política pública. Research, Society and Development, 9 (2).

Silva, E. S.,Junior, J. M.\&Suchara, E. P. (2018). Perfil de suicídios em município da Amazônia Legal. Cad. Saúde Colet, 26 (1), 84-91.

Silva, F. P.\& Souza, A. C. (2021). Atitudes dos profissionais no cuidado em situação de suicídio: estudo transversal. Online Braz J Nurs [Internet].https://docs.bvsalud.org/biblioref/2021/05/1222613/6418-pt.pdf

Silva, N. K. N., Carvalho, C. M. S., Magalhães, J. M., Júnior, J. A. M. C., Sousa, B. V. S \& Moreira, W. C. (2017). Ações do enfermeiro na atenção básica para prevenção do suicídio. Rev. Eletrônica Saúde Mental Álcool Drog, 13 (2),71-77.

Sousa, C.M.S., Mascarenhas, M. D. M., Malvina, K. R. O. G., Rodrigues, T. P., Miranda, C. E. S.\& Frota, K.M. G. (2020). Ideação suicida e fatores associados entre escolares adolescentes. Rev. Saude Publica, 54 (33). 
Research, Society and Development, v. 10, n. 10, e02101018547, 2021

(CC BY 4.0) | ISSN 2525-3409 | DOI: http://dx.doi.org/10.33448/rsd-v10i10.18547

Sousa, J. F., Sousa, V. C., Carvalho, C. M. S., Amorim, F. C. M., Fernandes, M. A., Coelho, M. C. V. S. \& Silva, J. S. (2019). Prevenção ao suicídio na atenção básica: concepção de enfermeiros. Rev. Cuid., 10 (2).

Stoppa, R. G., Wanderbroocke, A. C. N. S.\&Azevêdo, A. V. S. (2020). Profissionais de Saúde no Atendimento ao Usuário com Comportamento Suicida no Brasil: Revisão Sistemática. Revista Psicologia e Saúde, 12 (4).

Storino, B. D., Campos, C. F., Chicata, L. C. O., Campos, M. A., Matos, M. S. C., Nunes, R. M. C. M. \& Vidal, E. L. (2018). Atitudes de profissionais da saúde em relação ao comportamento suicida. Cad. saúde colet, 26(4).

Tadiello, J. M.\& Seixas, C.E. (2020).O perfil epidemiológico dos pacientes com risco suicida acolhidos no serviço de saúde Santa Maria acolhe. Rev. Psicol Saúde e Debate, 6 (1), 151-164.

Teixeira, S. M. O., Souza, L. E. C.\& Viana, L. M. M. (2018).O suicídio como questão de saúde pública. Rev Bras Promoç Saúde, 31(3).

WORLD HEALTH ORGANIZATION. (2014). Preventing suicide: a global imperative. Geneva: World Health Organization;https://apps.who.int/iris/bitstream/handle/10665/131056/9789241564779_eng.pdf?sequence=1 